\title{
Interaction between a cationic bolaamphiphile and DNA: the route towards nanovectors for oligonucleotide antimicrobials
}

\author{
Marianna Mamusa ${ }^{1}$, Claudio Resta ${ }^{1}$, Francesco Barbero ${ }^{2}$, Davide Carta $^{3,4}$, Doroty Codoni ${ }^{3,5}$, \\ Kostas Hatzixanthis ${ }^{3,6}$, Michael McArthur ${ }^{3,7}$, Debora Berti ${ }^{1, *}$
}

${ }^{1}$ Department of Chemistry “Ugo Schiff” and CSGI, University of Florence. Via della Lastruccia 3, 50019 Sesto Fiorentino (FI), Italy

${ }^{2}$ Nanovector s.r.1. Via Livorno 60, 10144 Torino, Italy

${ }^{3}$ Procarta Biosystems Ltd. Innovation Centre, Norwich Research Park, Norwich UK, NR4 7GJ

${ }^{4}$ Biological Chemistry Department, John Innes Centre, Norwich, NR4 7UH, UK

${ }^{5}$ Kuecept Ltd. 16/17 Station Close, Potters Bar, EN6 1TL, UK

${ }^{6}$ School of Pharmacy, University of East Anglia, Norwich, NR4 7TJ, UK

${ }^{7}$ Norwich Medical School, University of East Anglia, Norwich, NR4 7UQ, UK

* Corresponding Author: Prof. Debora Berti

e-mail: debora.berti@unifi.it, phone:+39055 4573038

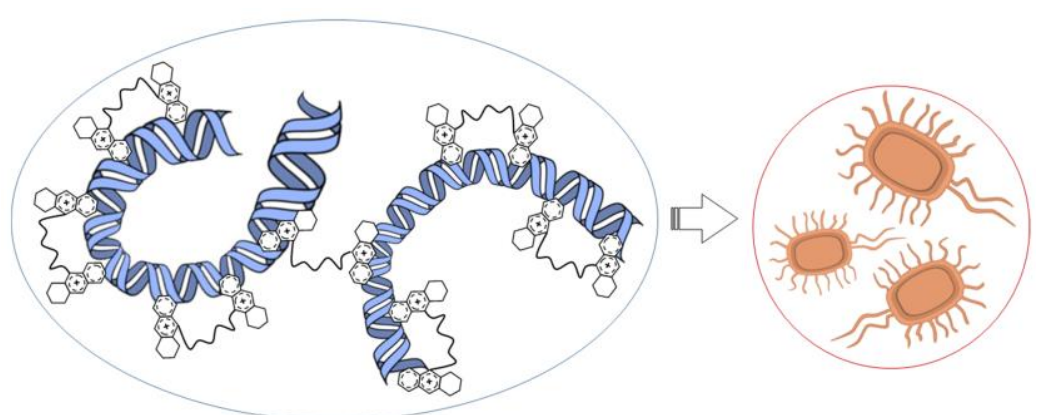

[12-bis-THA]Cl 2 complexes a Transcription Factor Decoy (TFD) to defeat infections from antibiotic-resistant bacteria 


\section{ABSTRACT}

Bacterial resistance to antimicrobials is a global threat that requires development of innovative therapeutics that circumvent its onset. The use of Transcription Factor Decoys (TFDs), DNA fragments that act by blocking essential transcription factors in microbes, represents a very promising approach. TFDs require appropriate carriers to protect them from degradation in biological fluids and transfect them through the bacterial cell wall into the cytoplasm, their site of action. Here we report on a bolaform cationic surfactant, [12-bis- $\mathrm{THA}] \mathrm{Cl}_{2}$, with proven transfection activity in vivo. By studying the physical-chemical properties of its aqueous solutions with light scattering, cryo-TEM, $\zeta$-potential, absorption and fluorescence spectroscopies, we prove that the bolaamphiphiles associate into transient vesicles which convert into one-dimensional elongated structures over time. These surfactant assemblies complex TFDs with extremely high efficiency, if compared to common cationic amphiphiles. At $\mathrm{Z}_{+/-}=10$, the nanoplexes are stable and have a size of $120 \mathrm{~nm}$, and they form independently of the original morphology of the [12-bisTHA $] \mathrm{Cl}_{2}$ aggregate. DNA is compacted in the nanoplexes, as shown through CD spectroscopy and fluorescence, but is readily released in its native form if sodium taurocholate is added.

\section{KEYWORDS}

Bacterial resistance; transcription factor decoy; DNA delivery; bolaamphiphile; circular dichroism; light scattering. 


\section{Introduction.}

The "golden age" of antimicrobial breakthrough lasted 60 years from the discovery of penicillin in 1928 and supplied the large majority of the drugs in current use [1]. However, bacteria naturally develop resistance, and contributory factors include: the limited number of therapeutic targets that antimicrobials act on; the greater need due to the demands of modern medicine and aging populations; their misuse and overuse; most importantly, the failure to find new antimicrobials to restock the pipeline [2]. Hence, antimicrobial resistance now constitutes a serious global threat [3]. Several alternative approaches to traditional small molecule discovery are being developed, such as bacteriophage therapy [4], antibodies [5], peptidomimetics [6] and nucleic acid therapies [7]. Previous work has demonstrated the efficacy of oligonucleotide Transcription Factor Decoys (TFDs) in controlling gene expression in both eukaryotic [8] and bacterial cells [9]. However,

oligonucleotide delivery faces an important challenge: how to transport an enzymatically labile, negatively charged molecule across biological fluids and through the bacterial cell wall.

The bacterial membrane is negatively charged due to the presence of phospholipids such as phosphatidylglycerol, cardiolipin, or phosphatidylserine [10]. The structural integrity of the membrane can be disrupted by initial electrostatic interaction using cationic lipids or surfactants. These are commonly employed as vectors for non-viral gene delivery for therapeutic purposes, as they are effective in the condensation and transfection of genetic material [11]. Among others, cationic bolaamphiphiles are attractive targets for the development of novel agents for nucleic acid delivery. They are a class of surfactants constituted by two functional hydrophilic headgroups linked by a hydrophobic moiety. Dequalinium, an approved antimicrobial, is a symmetric bolaform surfactant with two identical cationic headgroups. This molecule can complex plasmid DNA, 
protecting it from DNAse attack $[12,13]$ and transporting it into cellular mitochondria in vitro [14], which share some of the properties of bacterial cell walls.

In this work, we present a cationic bolaamphiphilic molecule, the symmetric 10,10 '-(dodecane1,12-diyl)-bis(9-amino-5,6,7,8-tetrahydroacridinium) chloride or [12-bis-THA]Cl 2 (Scheme 1), which has demonstrated the potential of delivering TFDs in pathogenic bacteria in animal models to block essential genes [15]. The mechanism of transfection by [12-bis- $\mathrm{THA} \mathrm{Cl}_{2}$ is being investigated but it is assumed that binding is driven by electrostatic interactions between the delocalized charge of the quaternary ammonium and the negatively charged components of the bacterial cell wall. In a similar manner, the interaction between the cationic bolaamphiphile and the negatively charged phosphate backbone of the oligonucleotide may lead to strong binding and condensation of the TFD, to render it resistant to nuclease digestion. Here we analyze the physicalchemical properties of an aqueous suspension of [12-bisTHA $] \mathrm{Cl}_{2}$ and its complex with a TFD to better understand its properties and inform strategies for its successful formulation.

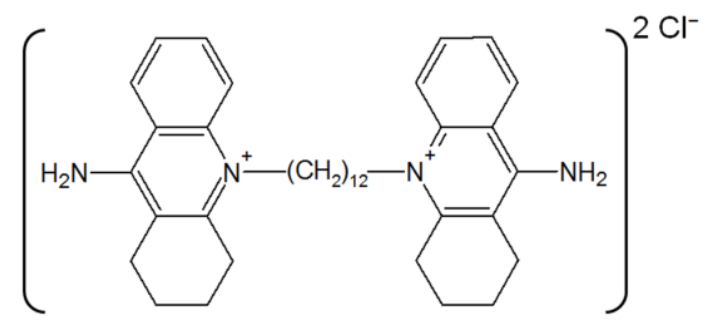

Scheme 1. Chemical structure of 10,10'-(dodecane-1,12-diyl)bis(9-amino-5,6,7,8tetrahydroacridinium) chloride, or [12-bis-THA $] \mathrm{Cl}_{2}$. 


\section{Experimental section.}

\section{Materials:}

[12-bis-THA $] \mathrm{I}_{2}$ was synthesized by Shanghai Chempartner Co., LTD. [12-bis-THA]Cl 2 was obtained by anion exchange of [12-bis-THA]I 2 (see details in Supporting Information, $\mathrm{SI}^{\S}$ ).

The TFD was manufactured and purified by HPLC at AxoLabs (Kulmbach, Germany). It consists of 77 base pairs deriving from the two following sequences: 5' CTT GGT TTT TCC AAG TAA TAC GAC AAA ACT AGT TAA ATT TCA TTG AAG GAA TAA AAA TAT AAT TAT AGA ATT GAT TA 3'; 5' TAA TCA ATT CTA TAA TTA TAT TTT TAT TCC TTC AAT GAA ATT TAA CTA GTT TTG TCG TAT TAC TTG GAA AAA CCA AG 3'. These oligonucleotides were suspended in water at concentrations of $510^{-7} \mathrm{~mol} / \mathrm{L}$, mixed in equal volumes and annealed by heating to $95^{\circ} \mathrm{C}$ for 2 minutes and then allowed to cool to room temperature. They were then ligated with T4 DNA ligase overnight at $16{ }^{\circ} \mathrm{C}$ to form a monomeric circle, containing the binding site for the transcription factor, and purified by ethanol precipitation. Unligated products were removed by an Exonuclease I digestion step following which the TFD was reprecipitated and its concentration adjusted to $1 \mathrm{mg} / \mathrm{mL}$.

Ultrapure water was obtained by means of a Millipore Elix ${ }^{\circledR} 3$ water purification system.

\section{Sample preparation:}

[12-bis-THA $] \mathrm{Cl}_{2}$ solutions were obtained by adding the powder in water and stirring by means of a vortex mixer (dilute solutions) or sonicating in an ultrasound bath (concentrated solutions). [12-bis-THA] $\mathrm{Cl}_{2} / \mathrm{TFD}$ nanoplexes were prepared by mixing the two aqueous solutions in the appropriate proportions. 


\section{Methods:}

Inductively-coupled plasma atomic emission spectroscopy (ICP-AES) analysis of the residual iodine content in [12-bis-THA $] \mathrm{Cl}_{2}$ was carried out using a Varian 720 -ES spectrophotometer (Melbourne, Australia).

Elemental analysis was performed using a CHN-S Flash E1112 Thermo Finnigan elemental analyzer.

Thermogravimetric Analysis (TGA) was performed on a TA Instruments SDT Q600 (New Castle, DE, USA). Between 4-7 mg of [12-bis-THA] $\mathrm{Cl}_{2}$ dry powder was weighed in an aluminum pan and heated up to $500{ }^{\circ} \mathrm{C}$ at $10{ }^{\circ} \mathrm{C} / \mathrm{min}$.

Surface tension measurements were carried out on a Teclis Instruments (formerly I.T. Concept, Longessaigne, France) dynamic tensiometer using the pendant drop method. All measurements were carried out at $25^{\circ} \mathrm{C}$.

Steady-state fluorescence of [12-bis-THA]Cl $\mathrm{C}_{2}$ solutions was measured on a LS50B spectrofluorometer (Perkin-Elmer, Italy). The spectra were recorded in the corrected mode, between 300 and $500 \mathrm{~nm}$, with an excitation wavelength of $244 \mathrm{~nm}$ and $2.5 \mathrm{~nm}$ slits. For each sample, 20 acquisitions were collected at $25^{\circ} \mathrm{C}$ and averaged.

Light Scattering (LS) experiments were performed on a Brookhaven BI9000-AT digital autocorrelator, equipped with a green laser $(\lambda=532 \mathrm{~nm}$; Torus, mpc3000, LaserQuantum, UK). The scattered intensity was collected at $90^{\circ}$, using a pinhole aperture of $200 \mu \mathrm{m}$. The samples were placed in glass tubes, which were immersed in a thermostatic cell filled with decahydronaphtalene to match the glass refractive index. The scattering intensity of pure toluene was used as a standard. In the Rayleigh-Gans-Debye regime [16], the intensity $I_{s}$ of the light scattered by a monodisperse 
colloidal solution is a function of the number concentration of particles, $c$, and the scattering vector $\vec{q}$. The modulus $q$ is equal to:

$q=\frac{4 \pi n_{s}}{\lambda} \sin \frac{\theta}{2}$

Eq. 1

with $\lambda$ being the wavelength of the incident light $I_{0}, n_{s}$ the refractive index of the medium, and $\theta$ the collection angle of the scattered light. As such, $I_{s}$ can be written:

$I_{S}(\vec{q}, c)=I_{0} \frac{f(\theta)}{R_{0}^{2}} c V_{S} \Delta \rho^{2} V_{P}^{2} P(\vec{q}) S(\vec{q}, c)$

Eq. 2

where $f(\theta)$ is a geometrical factor, $R_{0}$ the sample-to-detector distance, $V_{s}$ and $V_{P}$ the solvent and particle volumes respectively, and $\Delta \rho$ the "contrast" or difference of refractive indexes between particles and suspension medium. $P(\vec{q})$ and $S(\vec{q}, c)$ are, respectively, the form factor and the structure factor (the latter is equal to 1 in very dilute solutions). In a Dynamic Light Scattering (DLS) experiment, we exploit the time correlation function of the random fluctuations in scattered intensity due to Brownian motions in solution [17]. By fitting the autocorrelation function with the appropriate algorithms, one can derive the diffusion coefficient of the particles and, by assuming a spherical shape, their average hydrodynamic diameter $\left(D_{H}\right)$ through the Stokes-Einstein law:

$D_{0}=\frac{k_{B} T}{3 \pi \eta D_{H}}$

Eq. 3 
where $k_{B}$ is Boltzmann's constant, $T$ the absolute temperature, and $\eta$ the medium's viscosity. Depending on the samples, the fitting of the autocorrelation function of the scattered intensity at $90^{\circ}$ was performed either with the cumulant method [17] or by inverting the autocorrelation function with the CONTIN algorithm [18].

$\zeta$-potentials were obtained from electrophoretic mobility measurements, performed on a Brookhaven ZetaPALS (Phase Analysis Light Scattering) instrument, equipped with a laser operating at $659 \mathrm{~nm}$. The scattered intensity was collected at $15^{\circ}$ to determine the electrophoretic mobility; the $\zeta$-potentials were then calculated through the Helmholtz-Smoluchowski equation.

Cryogenic transmission electron microscopy (cryo-TEM) experiments were carried out at the Institut de Biologie Paris-Seine, Université Pierre et Marie Curie, Paris, France. The specimens were rapidly frozen by plunge-freezing in liquid ethane, cooled by liquid nitrogen (LEICA EM CPC, Wien, Austria). The cryofixed specimens were mounted into a Gatan cryoholder (Gatan inc., Warrendale, PA) for direct observation at $-180^{\circ} \mathrm{C}$ in a JEOL $2100 \mathrm{HC}$ cryo-TEM operating at 200 $\mathrm{kV}$ with a LaB6 filament. Images were recorded in zero-loss mode with a Gif Tridiem energyfiltered-CCD camera, equipped with a $2 \mathrm{k} \times 2 \mathrm{k}$ pixel-sized chip (Gatan Inc., Warrendale, PA). Acquisition was accomplished with the Digital Micrograph software (version 1.83.842, Gatan inc., Warrendale, PA).

Circular Dichroism (CD) measurements were performed using a Jasco J-715 spectropolarimeter. The solutions were contained in quartz cells with optical path lengths of $1 \mathrm{~cm}$ or $1 \mathrm{~mm}$ depending on the sample. CD spectra were recorded at room temperature in the $200-400 \mathrm{~nm}$ range.

$\mathrm{UV}$-vis spectra were acquired at $25{ }^{\circ} \mathrm{C}$ on a Varian Cary 100 Bio spectrophotometer, equipped with a Varian Cary temperature controller. 


\section{Results and Discussion.}

The compound [12-bis-THA $]^{2+}$ is a bolaform cationic surfactant of molecular formula $\mathrm{C}_{38} \mathrm{H}_{52} \mathrm{~N}_{4}$. The iodide salt is soluble in DMSO and methanol, and only sparingly in water. When DMSO solutions are dispersed in water by vigorous stirring, a suspension of crystallites is formed, which precipitates within some hours. On the other hand, the chloride salt (Scheme 1) shows higher water solubility, which in principle implies a higher bioactivity. For these reasons, the subject of this investigation was the chloride salt.

At $25{ }^{\circ} \mathrm{C}$ [12-bis-THA] $\mathrm{Cl}_{2}$ dissolves in water up to $1 \mathrm{mg} / \mathrm{mL}\left(1.610^{-3} \mathrm{~mol} / \mathrm{L}\right)$. Such difference in solubility with respect to the iodide salt is expected: indeed, ions with similar polarizabilities (e.g. two "soft" ions such as $[12-\mathrm{bis}-\mathrm{THA}]^{2+}$ and $\left.\mathrm{I}^{-}\right)$tend to form stronger ion pairs and are therefore less soluble salts. Examples of different phase behaviors arising from a simple counterion exchange are very common in colloid science: for instance, aqueous solutions of hexadecyltrimethylammonium bromide $(\mathrm{CTAB})$ and chloride (CTAC) are characterized by the presence of large elongated micelles in the former case and globular micelles in the latter [19], because of the higher association of the counterion with the cationic assemblies.

Below the solubility limit, the system appeared slightly opalescent as prepared, indicating the presence of self-assembly aggregates of some kind (large enough to scatter visible light). At higher concentrations, a saturated solution was obtained. The binary systems containing $80 \mathrm{wt} \%, 50 \mathrm{wt} \%$, $30 \mathrm{wt} \%$, and $20 \mathrm{wt} \%$ water showed no evidence of formation of liquid-crystalline phases. 


\section{Aqueous solution behavior of [12-bis-THA]Cl 2}

Bolaform surfactants are known to self-assemble in solution, leading to a wide range of aggregate morphologies depending on their chemical structure: tubules, fibers, ribbons, micelles and vesicles are among the common nano- and micrometric supramolecular structures reported for bolaamphiphiles [20]. Dynamic Light Scattering (DLS) was used here to infer the hydrodynamic size of [12-bis-THA]Cl $\mathrm{Cl}_{2}$ assemblies. For relatively concentrated dispersions $\left(1.010^{-3} \mathrm{~mol} / \mathrm{L}\right)$, the DLS analysis shown in Figure 1 yielded Z-average hydrodynamic sizes of $120 \pm 20 \mathrm{~nm}$, with elevated polydispersity $(\sim 30 \%)$.
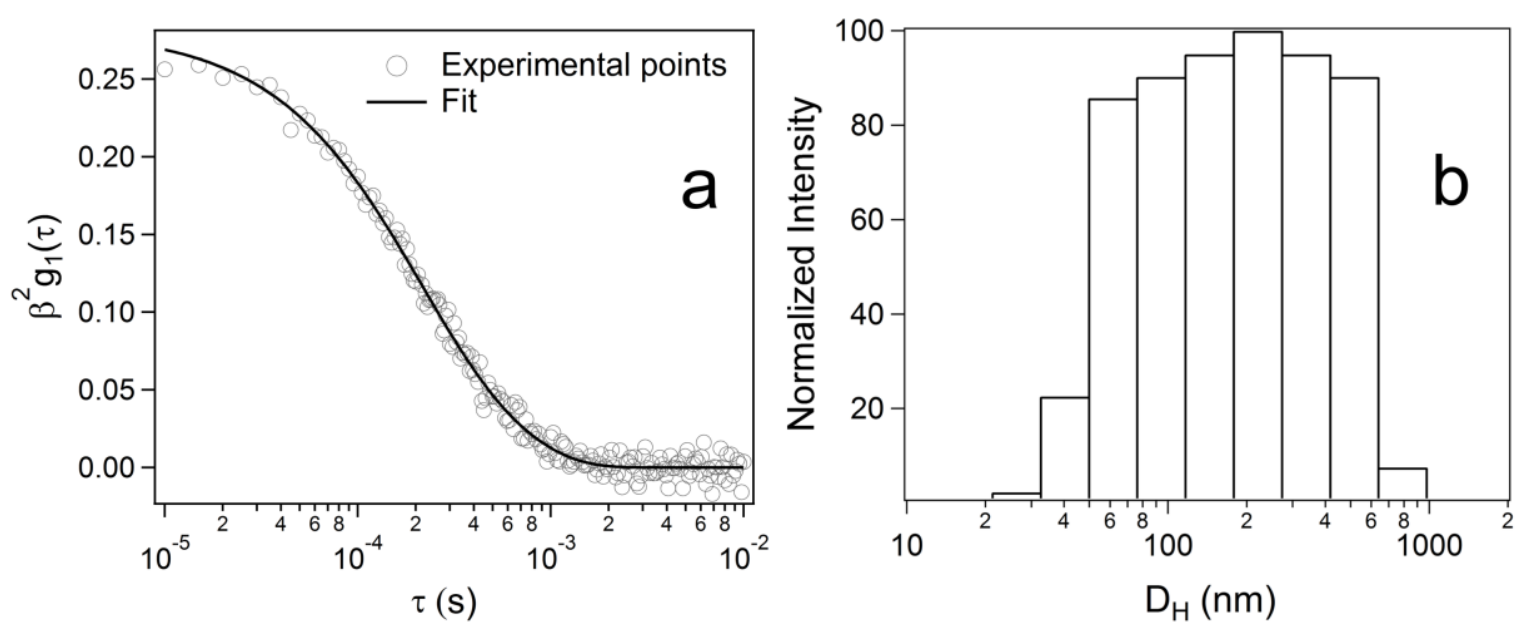

Figure 1. a) DLS autocorrelation function corresponding to a solution of $1.010^{-3} \mathrm{~mol} / \mathrm{L}$ [12-bisTHA $\mathrm{Cl}_{2}$, and fit of the experimental data with the cumulant method. b) Intensity-weighed hydrodynamic size distribution obtained by CONTIN analysis of a).

For samples more dilute than $4.510^{-4} \mathrm{~mol} / \mathrm{L}$, the scattering intensity was only slightly higher than that of pure water. This is likely due to a combination of low concentration and low contrast between the surfactant assemblies and water (see Equation 2). Therefore, the size and 
polydispersity of [12-bis-THA $] \mathrm{Cl}_{2}$ aggregates in dilute solutions cannot be determined by means of DLS. Similarly, the very low scattering invalidated any attempts to reliably measure the $\zeta$ potential of these colloidal objects. Even synchrotron small-angle x-ray scattering (SAXS) experiments on aqueous solutions of [12-bis- $\mathrm{THA}] \mathrm{Cl}_{2}$ at maximum solubility were unsuccessful, yielding spectra that were very similar to those of pure water. For these reasons we turned to cryogenic transmission electron microscopy (cryo-TEM) in order to determine the type of aggregates formed by [12-bis-THA]Cl 2 . Imaging of a freshly prepared solution $\left(1.810^{-4} \mathrm{~mol} / \mathrm{L}\right.$, Figure 2) showed that this surfactant forms a mixture of elongated colloidal structures and (most probably monolayer) vesicular assemblies termed bolasomes. The former appear to be short needle-like objects [21], while the latter have irregular shapes and diameters around or below 100 $\mathrm{nm}$, and they appear sometimes aggregated in groups of 2-3 vesicles. Despite the difference in [12bis-THA $] \mathrm{Cl}_{2}$ concentration, this compares well with the DLS analysis. Similarly, in a work involving aqueous solutions of Dequalinium, a cationic bolaamphiphile with a very similar chemical structure to [12-bis-THA] $\mathrm{Cl}_{2}$, the authors observed bolasomes with sizes of $300 \mathrm{~nm}$ (determined by negative-staining TEM) [12]. 


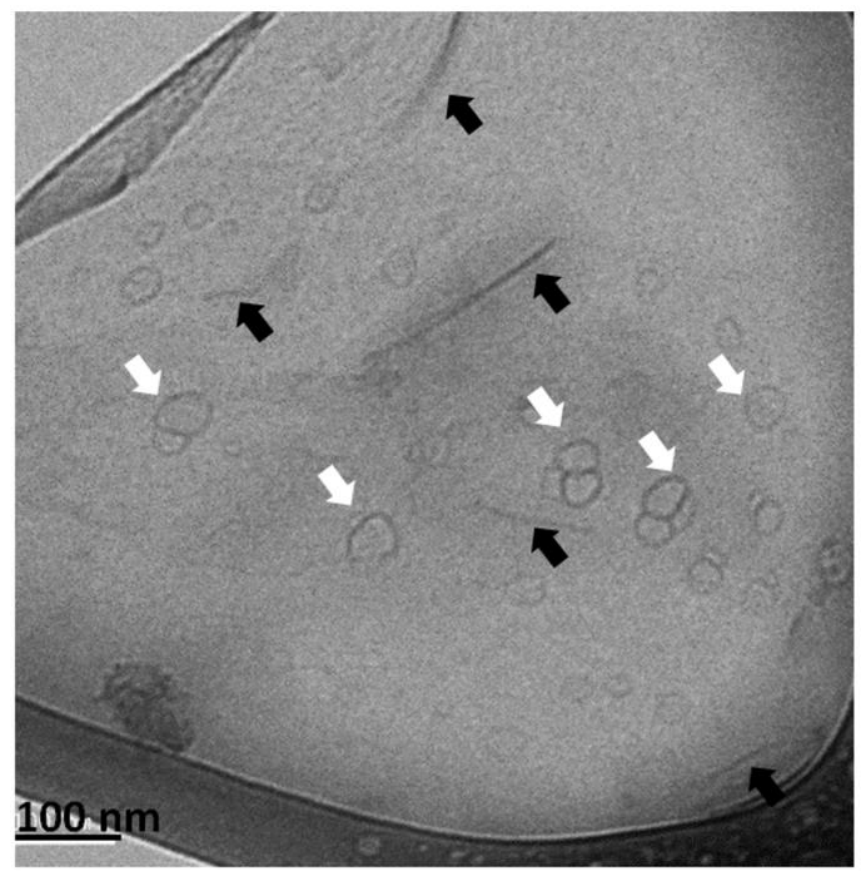

Figure 2. Cryo-TEM image of an aqueous solution of [12-bis- $\mathrm{THA}] \mathrm{Cl}_{2}, 1.810^{-4} \mathrm{~mol} / \mathrm{L}$, as prepared. White arrows: vesicular structures; black arrows: needle-like structures.

A precise knowledge of the threshold concentration for aggregation is of primary importance to formulate amphiphilic drugs in water or physiologically relevant media; therefore, we focused our efforts in finding the onset concentration for the formation of [12-bis-THA]Cl 2 aggregates. Previous literature on symmetric bolaform surfactants demonstrates that very similar thermodynamic principles of self-assembly apply as for classic surfactants [22]. However, the driving force for aggregation is lower than for the corresponding single-headed amphiphiles, due to the presence of the second polar headgroup, and this leads to generally higher critical aggregate concentrations (CAC). We attempted to determine a $\mathrm{CAC}$ for [12-bis- $\mathrm{THA}] \mathrm{Cl}_{2}$ in water by tensiometry with the pendant drop method. The surface tension $v$ s. time curves showed a monotonically decreasing trend without reaching the expected equilibrium plateau (see Figure S2, $\mathrm{SI}^{\S}$ ). One tentative explanation might be found by looking more closely at the structure of the 
bolaamphiphile: the positive charge of the headgroups is mostly localized on the central aminopyridinium ring, while the benzene and cycloalkyl groups are less polar. In addition, the alkyl chains connecting the two headgroups should kink to accommodate both polar ends into the water subphase. Likely, the adsorption at the interface in the energetically favored conformation is a slow process, if compared to traditional single-chained surfactants. While a clear drop in surface tension is observed, its equilibrium value could not be appreciated in our experimental time frame (up to several hours).

As mentioned, the very low scattered intensity even in relatively concentrated samples prevents a reliable determination of the $\mathrm{CAC}$ with static light scattering. Also, the traditional method involving pyrene as a fluorescent probe for hydrophobic environment [23] could not be used, due to mutual interference between pyrene and [12-bis-THA]Cl 2 fluorescence.

Indeed, [12-bis-THA $] \mathrm{Cl}_{2}$ shows absorption and fluorescence behavior due to its quinolinium subunits [24]. The absorption and fluorescence of aqueous solutions of [12-bis- $\mathrm{THA}] \mathrm{Cl}_{2}$ at different concentrations, ranging from $8.010^{-6} \mathrm{~mol} / \mathrm{L}$ to $1.610^{-3} \mathrm{~mol} / \mathrm{L}$, were measured and analyzed (Figures S3-S4, SI ${ }^{\S}$ ). The fluorescence spectra were characterized by a relatively broad band centered at $373 \mathrm{~nm}$, the intensity of which linearly increased with growing surfactant concentration until $4.010^{-5} \mathrm{~mol} / \mathrm{L}$ and reached its maximum at $8.010^{-5} \mathrm{~mol} / \mathrm{L}$ (Figure 3). This behavior is consistent with the increase in concentration of isolated fluorophores (surfactant monomers). For higher concentrations, the linearity was lost and the intensity began decreasing. This intensity decrease could be an indication of the self-assembly of [12-bis-THA] $\mathrm{Cl}_{2}$, where the close proximity between fluorophores in the ground state results in a significant fluorescence selfquenching. A data analysis through a classic Stern-Volmer approach [25] could provide important information about this concentration-dependent behavior. However, in our experimental 
conditions, the absorption properties of [12-bis-THA] $\mathrm{Cl}_{2}$ gave also rise to a strong primary inner filter effect, which contributed to the concentration-dependent decrease of fluorescence intensity. The contributions from the two phenomena are difficult to separate, making the CAC determination through the Stern-Volmer approach very complicated [25].

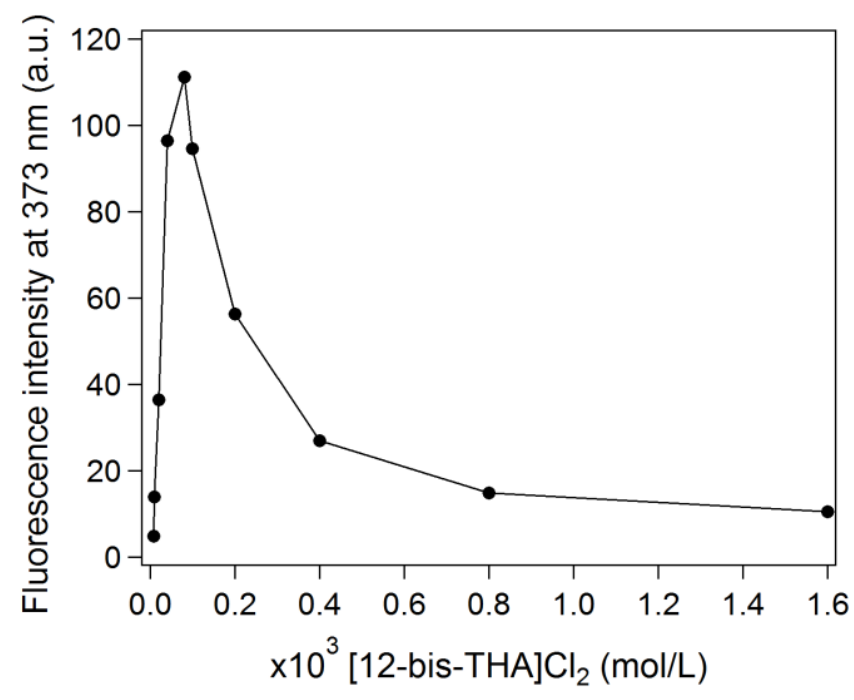

Figure 3. Steady state fluorescence intensity at the emission maximum $(373 \mathrm{~nm})$ as a function of [12-bis-THA $] \mathrm{Cl}_{2}$ concentration in water.

An analysis of previous literature shows that in some instances it is not possible to determine a critical micellar concentration (cmc) or a CAC for bolaamphiphilic molecules in solution [26,27]. In the case of Dequalinium, isothermic titration calorimetry, laser light scattering and Monte Carlo simulations cast doubts on the existence of a clear-cut monomer-to-aggregate transition [28]. By comparing our experimental results with the existing literature, we could not identify any clearcut aggregation threshold, which can be outside the concentration range accessible with our experimental techniques. Alternatively, the surfactant may aggregate in oligomers undetectable 
with DLS: in this case, aggregation would not proceed through pseudophase separation, but would rather be a stepwise phenomenon.

The slow equilibration processes of $[12-\text { bis-THA }]^{2+}$ at the air/water interface prompted us to monitor a possible time evolution of the size of the self-assemblies in solution. Light Scattering (LS) measurements were performed on solutions at different concentrations $\left(9.010^{-4}, 4.510^{-4}, 3.0\right.$ $10^{-4}$, and $\left.1.510^{-4} \mathrm{~mol} / \mathrm{L}\right)$ and temperatures $\left(4{ }^{\circ} \mathrm{C}, 25^{\circ} \mathrm{C}\right.$ and $\left.37^{\circ} \mathrm{C}\right)$. LS experiments were performed right after the preparation of the samples $(t=0)$ and 48 hours later $(t=48 \mathrm{~h})$; the results are presented in Figure 4.
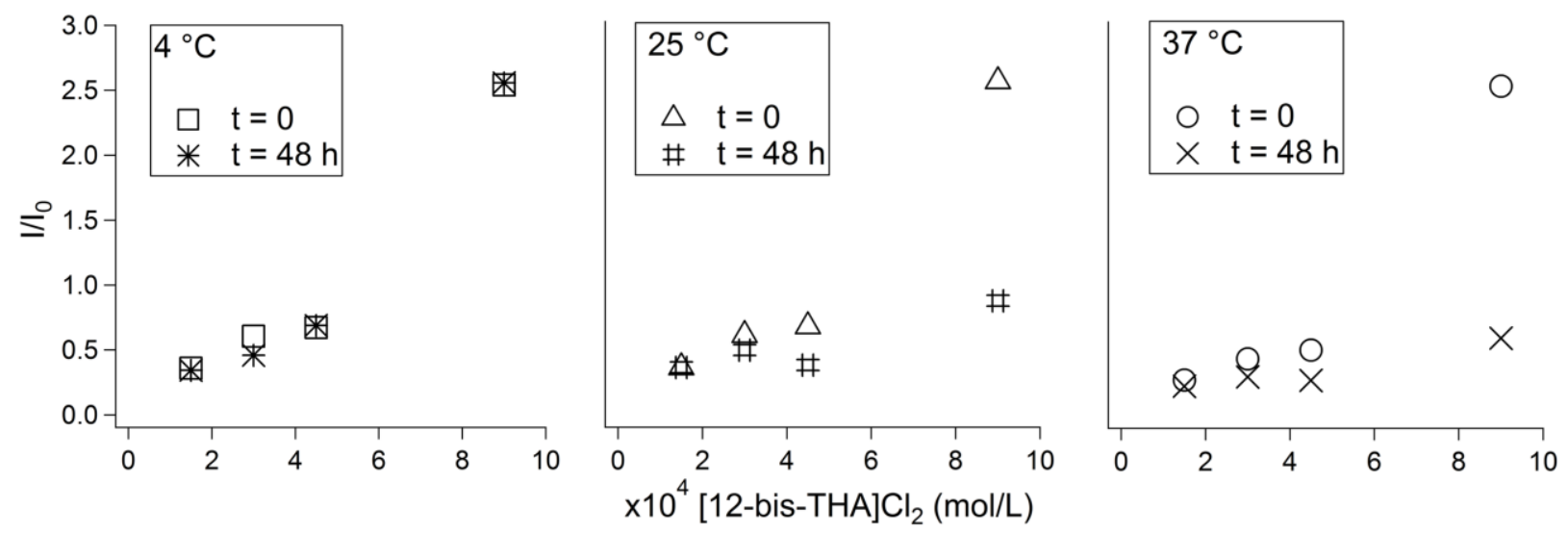

Figure 4. Normalized scattering intensity $\left(\mathrm{I} / \mathrm{I}_{0}\right.$, where $\mathrm{I}=$ sample and $\mathrm{I}_{0}=$ toluene $)$ of aqueous solutions of [12-bis-THA]Cl${ }_{2}$.

By comparing the $\mathrm{I} / \mathrm{I}_{0}$ values at $\mathrm{t}=0$ and $\mathrm{t}=48 \mathrm{~h}$ for the three batches of samples, it can be noticed that the solutions stored at $4{ }^{\circ} \mathrm{C}$ retained the scattering intensity observed immediately after preparation (even 7 days later, data not shown). The solutions stored at higher temperatures (25 ${ }^{\circ} \mathrm{C}$ and $37^{\circ} \mathrm{C}$ ) underwent a dramatic reduction of intensity over time. After ruling out the presence of a precipitate, the analysis of the autocorrelation functions revealed a decrease of the average 
size. For example, the mean hydrodynamic diameter of the aggregates in a $9.010^{-4} \mathrm{~mol} / \mathrm{L}$ solution stored at $25{ }^{\circ} \mathrm{C}$ was reduced by $50 \%$ over 24 hours, and a new population of scattering objects appeared after 5 days with a diameter below $10 \mathrm{~nm}$ (Figures S5-S6, SI ${ }^{\S}$ ). On the other hand, the sample stored at $4{ }^{\circ} \mathrm{C}$ retained the same average size. We verified the irreversibility of this phenomenon by cooling to $4{ }^{\circ} \mathrm{C}$ the samples that had been stored at $25^{\circ} \mathrm{C}$ and $37{ }^{\circ} \mathrm{C}$, and by stirring with a vortex mixer: in neither case did the systems revert to their initial state.

Since we could rule out precipitation, chemical reactions and molecular cleavage by means of UV-Vis and ${ }^{1} \mathrm{H}$ NMR analyses (data not shown), the phenomenon originating the loss of scattered intensity and the reduction of the apparent hydrodynamic diameters calculated via the StokesEinstein equation must be a morphological transformation of the colloidal assemblies. The intensity of light scattering scales with the squares volume of the scattering objects (Equation 2), so that large colloidal aggregates contribute a correspondingly higher scattering intensity. Also, we recall that cryo-TEM imaging (Figure 2) evidenced the co-existence of elongated objects, probably fibers, with bolasomes. The hydrodynamic size of a rod-like structure can be calculated according to Equation 4:

$R_{H}=\frac{L}{2 s-0.19-\frac{8.24}{s}+\frac{12}{s^{2}}}$

Eq. 4

where $L=$ length of the rod and $s=\ln (L / r)$, with $r=$ radius of the rod [29]. Assuming for example a rigid rod of about $100 \mathrm{~nm}$ length (such as those observed in Figure 2) and $1 \mathrm{~nm}$ radius (compatible with [12-bis-THA] $\mathrm{Cl}_{2}$ 's molecular size), we obtain a hydrodynamic diameter $\mathrm{D}_{\mathrm{H}} \approx 26 \mathrm{~nm}$. If the bolasomes were transient aggregates formed by the bolaamphiphile upon dissolution, converting entirely into fibers over time, this would account for the lower scattering intensity and smaller 
calculated size. Such transformation is clearly of spontaneous nature, as it proved to be irreversible: this evidence strongly hints at the fact that the final morphology represents the state of thermodynamic equilibrium of the system.

Turning to geometrical considerations, the type and shape of self-assembly aggregates in solution can be predicted by the packing parameter of the amphiphile: $P=V l_{c} / A_{0}$, where $V$ is the volume of the hydrophobic chain, $l_{c}$ its length, and $A_{0}$ the polar headgroup area [30]. In particular, vesicles will form for $1 / 2<P<1$, while $P \approx 1$ leads to infinite flat bilayers. A symmetrical bilayer does not have a spontaneous curvature, therefore the existence of vesicles is thermodynamically unfavorable for a binary system [31]. Nevertheless, exceptions to this rule exist. Let us consider a classic example among single-headed surfactants: NaAOT is characterized by $P \approx 1$ and it forms lamellar and bicontinuous (locally flat) cubic phases in water. However it also forms stable unilamellar vesicles at very low surfactant concentration $\left(\mathrm{CVC}=7.810^{-3} \mathrm{~mol} / \mathrm{L}\right)[32]$, where curved vesicles are thermodynamically more stable than isolated bilayer stacks. In this case, the curvature is allowed thanks to the bilayer asymmetry achieved by packing a lower number of molecules in the inner leaflet of the vesicle [31]. On the other hand, the symmetrical shape of the [12-bis-THA $] \mathrm{Cl}_{2}$ molecule would suggest elongated structures such as fibers or ribbons rather than spherical vesicles. The vesicular aggregates that form with an energy input, such as vortexing or sonicating to facilitate dissolution, are therefore short-lived and the bolasomes observed with cryoTEM in a freshly prepared solution of [12-bis-THA] $\mathrm{Cl}_{2}$ are irreversibly destroyed over time to form fibers (Scheme 2). In the abovementioned case of Dequalinium, where bolasomes were observed, no temporal evolution of these structures was reported by the authors.

The [12-bis-THA $] \mathrm{Cl}_{2}$ bolasomes are nevertheless metastable at $4{ }^{\circ} \mathrm{C}$, when thermal fluctuations are probably not enough to disrupt any intermolecular forces, maybe comprising weak $\pi-\pi$ 
interactions between the aromatic rings, which contribute to the bolasome assemblies. Therefore, by playing with storage temperature, we are able to control the morphology of [12-bis-THA] $\mathrm{Cl}_{2}$ aggregates.

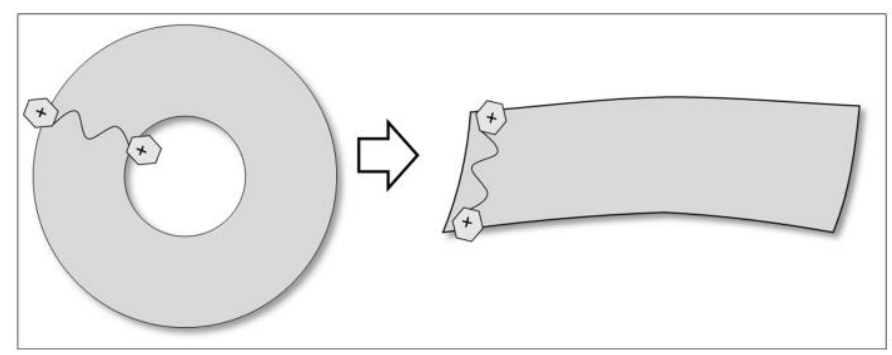

Scheme 2. Representation of the transition from bolasomes to one-dimensional elongated structures (fibers).

\section{Interaction of [12-bis-THA]Cl $\mathrm{Cl}_{2}$ with $\mathrm{DNA}$}

As mentioned, [12-bis-THA] $\mathrm{Cl}_{2}$ is mixed with an oligonucleotide transcription factor decoy to form a novel type of antimicrobial; therefore, the interaction between the bolaamphiphile and DNA was studied using a model TFD constituted by a double-stranded oligonucleotide, composed of 77 base pairs and especially rich in thymine and adenine.

Aqueous solutions of this TFD and [12-bis-THA] $\mathrm{Cl}_{2}$ were mixed in proportions leading to a positive-to-negative charge ratio $\mathrm{Z}_{+/-}=11$ (with the bolaamphiphile at $1.810^{-4} \mathrm{~mol} / \mathrm{L}$ ). The dispersion became opalescent, and LS experiments showed a scattered intensity about 20 times higher than for the neat bolaamphiphile. DLS analysis confirmed the presence of aggregates in suspension, with hydrodynamic sizes of $150 \pm 20 \mathrm{~nm}$ and about $20 \%$ polydispersity. A cryo-TEM image of these aggregates is shown in Figure S7, SI ${ }^{\S}$. The $\zeta$-potentials were in the order of $30 \pm 2$ 
$\mathrm{mV}$, in agreement with the excess of positively charged species. These experimental findings suggest complexation of the TFD by [12-bis-THA] $\mathrm{Cl}_{2}$, as expected by charge compensation considerations and by the vast body of literature about the formation of nanoplexes, i.e. amphiphile-DNA complexes, when DNA is added to a cationic surfactant solution. By maintaining an excess of positive charge, the TFD complexation would be complete, and the interaction of the nanoplex with the bacterial membranes, rich in negatively charged lipids, would be favored.

Importantly, the same type of aggregates were obtained regardless of the "age" of the initial [12bis-THA]Cl 2 solution, as shown by DLS analysis in Figure $\mathrm{S} 8, \mathrm{SI}^{\S}$. This proves that the properties of the [12-bis-THA $] \mathrm{Cl}_{2} / \mathrm{TFD}$ complex are independent on the morphology of the initial bolaamphiphile assemblies. From an applicative standpoint, this peculiarity could represent a great advantage in the design of the final formulations to be used as antibacterial agents, even though other variables, such as time and temperature stability of the complex, necessitate further evaluation.

When DNA forms complexes upon interaction with cationic surfactants, it assumes a condensed state and loses its chiral secondary structure. In this case, the interaction between nucleic acids and the cationic surfactant can be evaluated using CD spectroscopy, that exploits the differential absorption of left- and right-circularly polarized electromagnetic radiation by a sample [33]. The TFD, dissolved in ultrapure water at the concentration of $90 \mu \mathrm{g} / \mathrm{mL}$ (corresponding to $1.910^{-6}$ $\mathrm{mol} / \mathrm{L}$ ), was titrated with the aqueous bolaamphiphile solution up to and above the isoelectric point. The [12-bis-THA] $\mathrm{Cl}_{2}$ concentration in solution varied between 0 and $1.710^{-4} \mathrm{~mol} / \mathrm{L}$, corresponding to $\mathrm{Z}_{+/-}$variation from 0 to 1.8 . These values are far from the $\mathrm{Z}_{+/-}=11$ used in the earlier tests, since we are now looking at the transformations occurring near the isoelectric point. 
Figure 5a reports five representative spectra obtained in this titration experiment (the entire set of data and the corresponding absorption spectra are available in Figures $\mathrm{S} 9-\mathrm{S} 10, \mathrm{SI}^{\S}$ ).
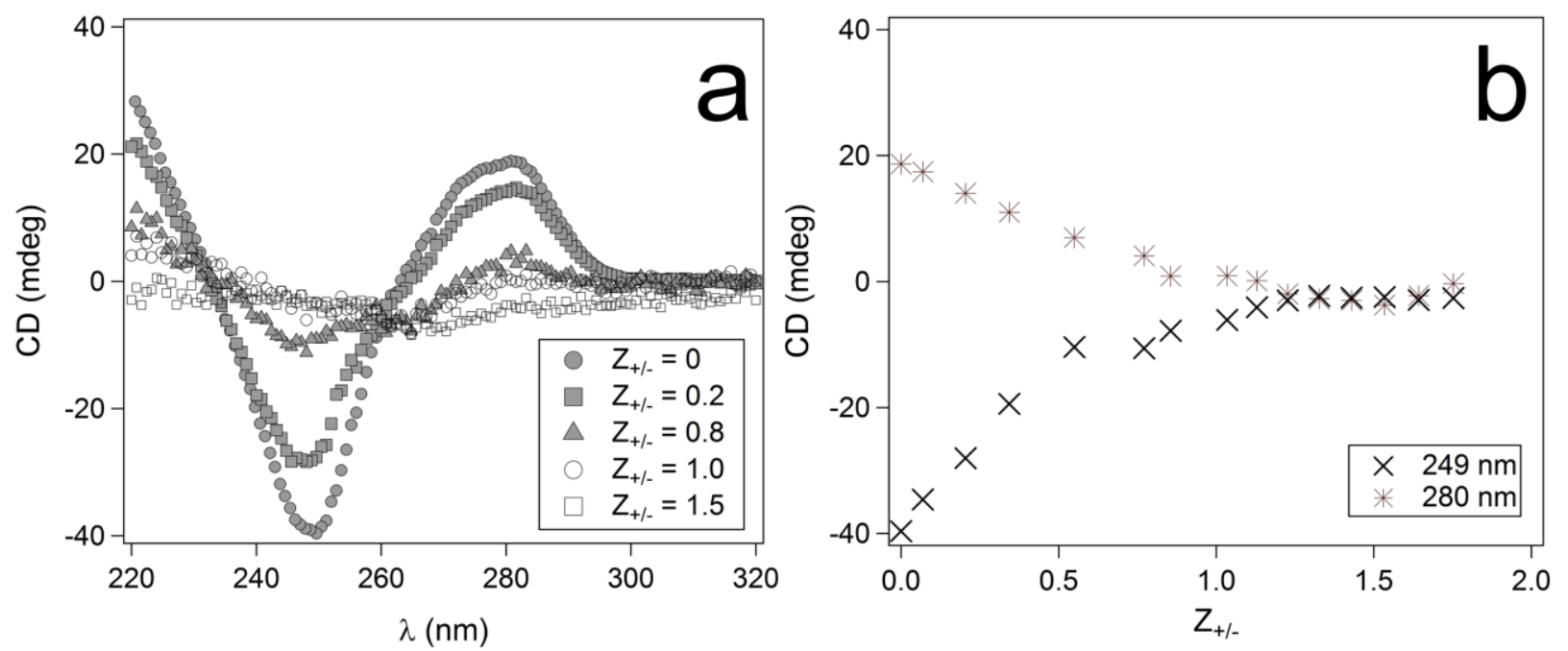

Figure 5. Titration of a 77-bp TFD (90 $\mu \mathrm{g} / \mathrm{mL}$, or $\left.1.910^{-6} \mathrm{~mol} / \mathrm{L}\right)$ with [12-bis-THA]Cl ${ }_{2}$ a) $\mathrm{CD}$ spectra for five significative positive-to-negative charge ratios $\left(\mathrm{Z}_{+/ /}\right)$. For the sake of clarity, 1 in every 8 points were traced. b) Plot of the CD values at 249 and $280 \mathrm{~nm}$, from spectra in Figure S9, as a function of $Z_{+/ .}$

The initial shape of the CD spectrum is characteristic of DNA in B-conformation: it presents a positive peak at $280 \mathrm{~nm}$, originating from base stacking, and a negative peak at $249 \mathrm{~nm}$, due to the helicity of the double strand. In particular, the negative band is quite intense, as typical of chiral DNA strands with high A+T base content [33]. The CD signal of the TFD starts changing upon the very first addition of [12-bis-THA] $\mathrm{Cl}_{2}$, as the two oppositely charged species interact in solution. The $\mathrm{CD}$ spectrum varies slightly even above the isoelectric point $\left(\mathrm{Z}_{+/-}=1.0\right)$; at $\mathrm{Z}_{+/-}=$ 1.5 , the bands of the original spectrum have completely flattened out, and further addition of surfactant does not lead to any change. This evidence is summarized in Figure 5b, where the trend 
of the CD is plotted, for the wavelengths $249 \mathrm{~nm}$ and $280 \mathrm{~nm}$, as a function of $Z_{+/ .}$. It is quite evident how the intensity reaches zero just above the isoelectric point. The same experimental trend has been evidenced for the condensation of different types of DNA by traditional [34] and gemini cationic surfactants $[35,36]$.

By observing the absorption spectra in Figure S10, one can notice the condensation of the oligonucleotide into packed complexes as the concentration of bolaamphiphile increases: the higher baseline is a clear signature of the solution's growing opalescence due to the presence of colloidal aggregates. This behavior can be represented by the plot of the absorbance at $400 \mathrm{~nm}$ as a function of [12-bis-THA] $\mathrm{Cl}_{2}$ concentration (Figure 6).

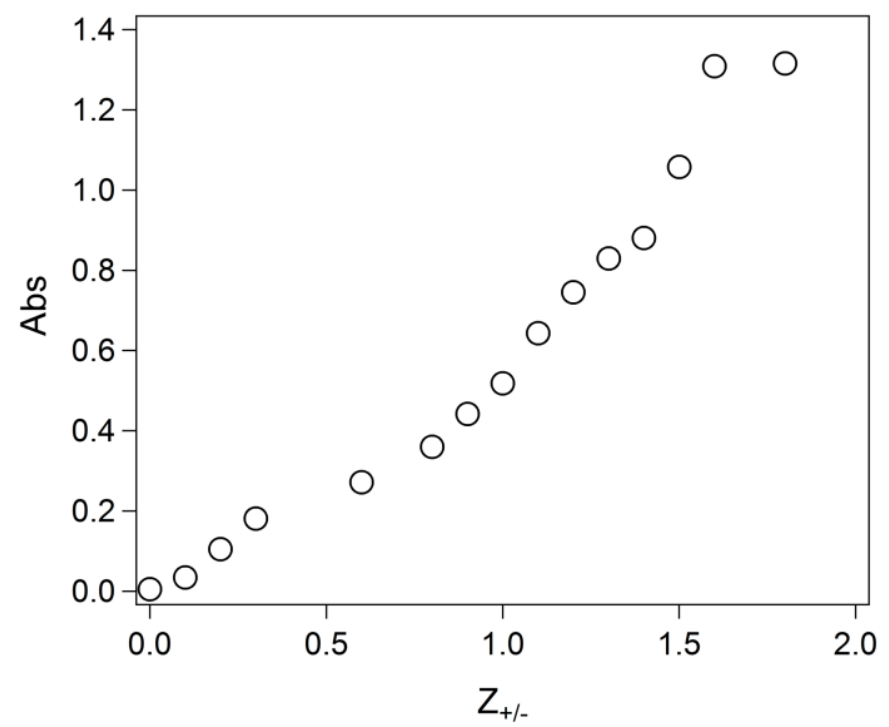

Figure 6. Plot of the absorbance values at $400 \mathrm{~nm}$ from the absorption spectra in Figure $\mathrm{S} 10$ as a function of $Z_{+/- \text {. }}$

To corroborate these results, we monitored the change in fluorescence intensity of [12-bisTHA $] \mathrm{Cl}_{2}$ upon titration with the TFD. The results are presented in Figure 7 as a function of $1 / \mathrm{Z}_{+/-}$ 
. Starting from a large excess of positive charge $\left(\mathrm{Z}_{+/-}=17\right.$, or $1 / \mathrm{Z}_{+/-}=0.05$ in Figure 7$)$, we witnessed an almost linear increase of fluorescence intensity up to a plateau; in correspondence of $\mathrm{Z}_{+/-}=1$, the fluorescence was quenched and kept decreasing with further increase of TFD. These results are in good agreement with those obtained by CD spectroscopy.

The electrostatic interaction between the oppositely charged species is the main driving force leading to the $1: 1$ complex between [12-bis-THA] $\mathrm{Cl}_{2}$ and DNA, similarly to what found by other authors for classic cationic surfactants [37], gemini surfactants [35,36], and bolaamphiphiles $[38,39]$. This can be an advantage compared to certain cationic species which necessitate higher

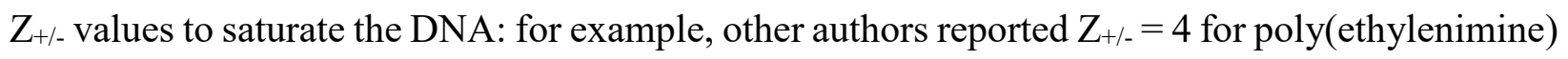
[40], $Z_{+/-}=10$ for TTAB and $Z_{+/-}=30$ for DTAB [34], which necessitate higher amounts of typically cytotoxic compounds to reach full condensation. To account for this higher binding efficiency, it is well known that planar aromatic and heterocyclic compounds, even uncharged, can interact with DNA thanks to different types of interactions: in a groove-bound fashion (a mix of hydrophobic, electrostatic, and hydrogen-bonding interactions), or by intercalation between the DNA base pairs [41]. In our case, [12-bis-THA]Cl 2 presents two positively charged condensed heterocyclic headgroups, each carrying an amine substituent: it is likely that DNA complexation by this bolaamphiphile results from several forces at play. 


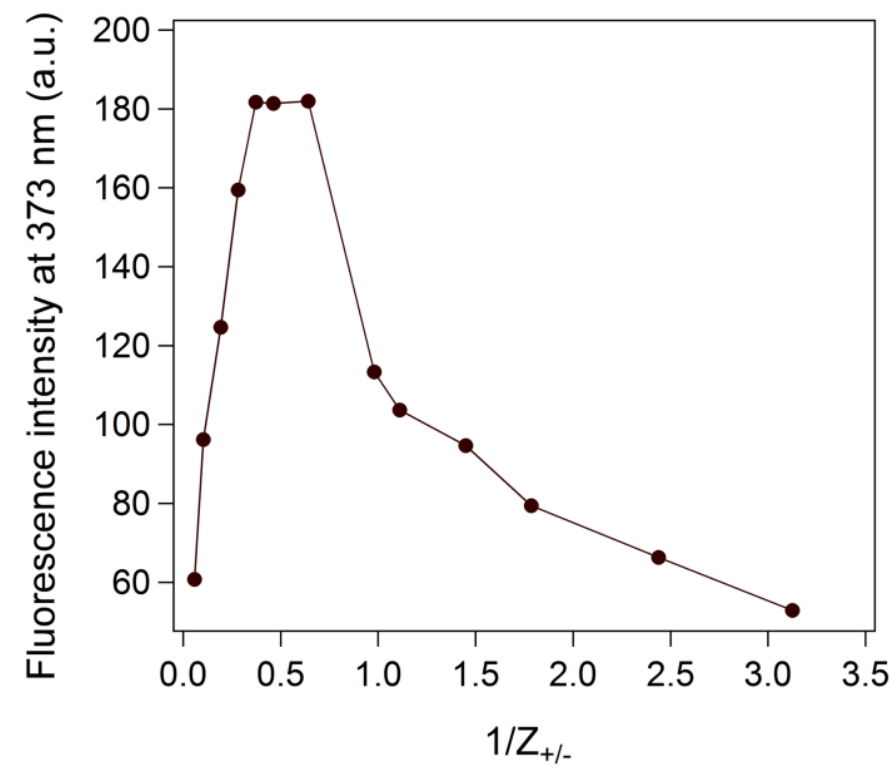

Figure 7. Plot of the fluorescence intensity at $373 \mathrm{~nm}$ as a function of the reverse of the positiveto-negative charge ratio, $1 / Z_{+/ .}$. Here $1 / Z_{+/-}$was used instead of $Z_{+/-}$to better render the trend of the fluorescence intensity over the course of the titration.

When DNA is packed into nanoplexes, it assumes a condensed state similar to denaturation. However, for the TFD to perform its therapeutic action, it needs to be renatured. In other words, the cationic carrier must release its DNA payload at the desired delivery site and in a biologically active form. Here, we show that the TFD/[12-bis-THA] $\mathrm{Cl}_{2}$ complex is reversible by breaking it with sodium taurocholate (NaTC), a common detergent used for the solubilization of lipids and previously employed for the dissociation of DNA/cationic lipid complexes [42]. Indeed, due to its negative charge, NaTC competes with the TFD's polyanionic backbone for interaction with [12bis-THA] $\mathrm{Cl}_{2}$.

The initial aqueous system contained the complex at $\mathrm{Z}_{+/-}=1.5\left([12\right.$-bis-THA $] \mathrm{Cl}_{2}: 2.510^{-4}$ $\mathrm{mol} / \mathrm{L}$ ), a condition in which the bolaamphiphile is in excess and the oligonucleotide is completely condensed, as demonstrated earlier in this work. The complex was titrated with aliquots of NaTC, 
and the resulting $\mathrm{CD}$ and UV spectra are presented in $\mathrm{SI}^{\S}$ (Figures S11-S12). At low NaTC concentration, the CD spectra are flat, meaning that the detergent is not enough concentrated to displace the TFD/bolaamphiphile complex. The first spectrum where the CD signal starts showing the features of the oligonucleotide is the one for $[\mathrm{NaTC}]=1.410^{-2} \mathrm{~mol} / \mathrm{L}$. From this point on, the intensity of the bands with maxima at $248 \mathrm{~nm}$ and $278 \mathrm{~nm}$ increases; the maximum amplitude is found at $[\mathrm{NaTC}]=4.210^{-2} \mathrm{~mol} / \mathrm{L}$, and further additions of detergent do not produce any variation. These trends are plotted in Figure 8b, displaying the CD at 248 and $278 \mathrm{~nm}$. The results just described are summarized in Figure 8a, which compares the CD spectra for the following representative samples: free TFD; condensed TFD when $\mathrm{Z}_{+/-}=1.5$; the $\mathrm{Z}_{+/-}=1.5$ complex in the presence of $7.010^{-2} \mathrm{~mol} / \mathrm{L} \mathrm{NaTC}$; free TFD with the same amount of NaTC (but no bolaamphiphile). This figure clearly evidences how the TFD/[12-bis-THA $] \mathrm{Cl}_{2}$ complex is broken in the presence of taurocholate, and the free renatured TFD is completely recovered.
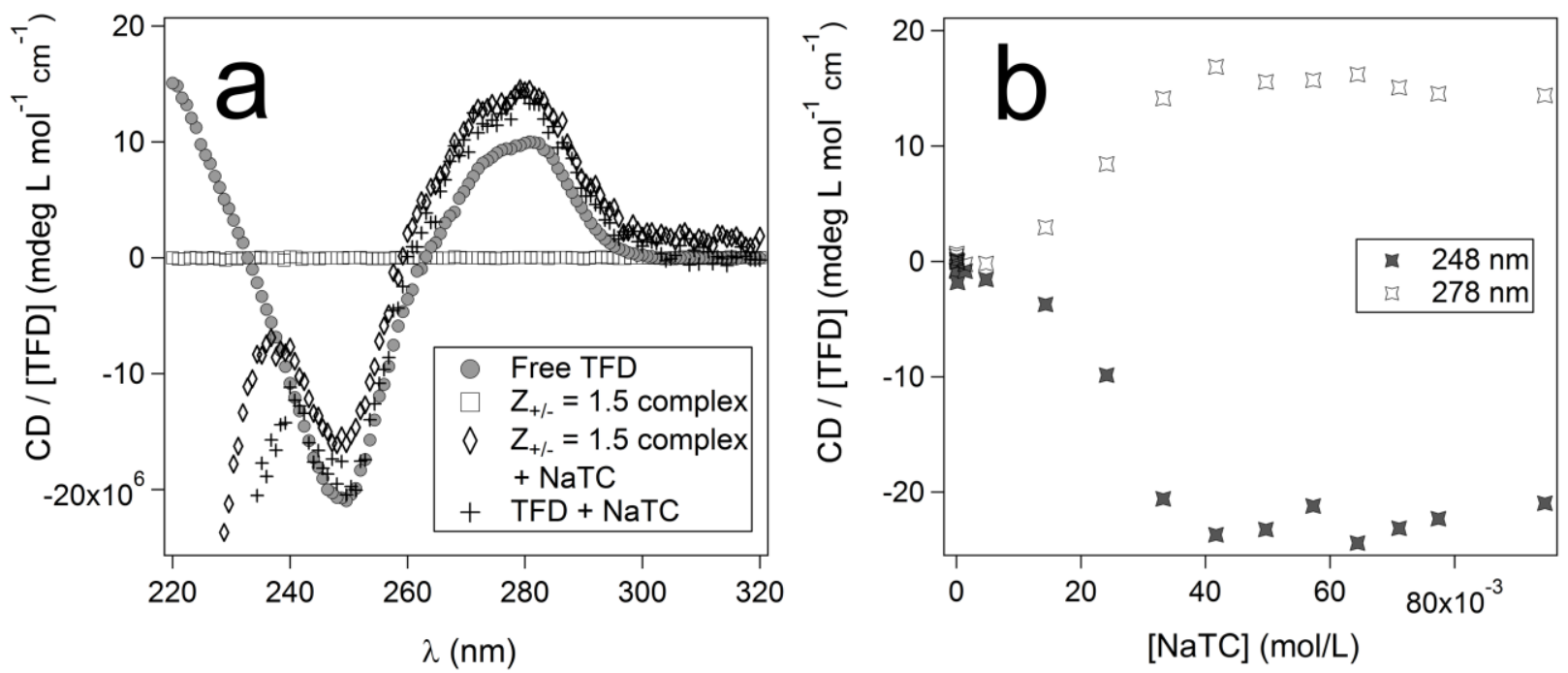

Figure 8. a) CD spectra showing the disruption of the 1:1.5 complex between the TFD and [12bis-THA] $\mathrm{Cl}_{2}$ using sodium taurocholate (NaTC, $\left.7.110^{-2} \mathrm{~mol} / \mathrm{L}\right)$. All spectra are normalized by the 
concentration of TFD. For the sake of clarity, 1 in every 8 points was traced for each spectrum. $b$ ) Plot of the CD values from spectra in Figure S11 at $248 \mathrm{~nm}$ and $278 \mathrm{~nm} v s$. NaTC concentration.

These results show that the therapeutically active TFD can be released from the complex. Such information is important in view of the biomedical applications of the TFD/[12-bis-THA $] \mathrm{Cl}_{2}$ complex. The release mechanism we described in the presence of a simple molecule as NaTC should be adapted to the conditions found in the bacterial cytoplasm. For example, the complex could be formulated in a stimuli-responsive fashion (e.g. to $\mathrm{pH}$, redox potential, etc.) in order to free the TFD only once it has crossed the bacterial cell wall. Further studies are underway to better define the conditions of existence of this complex and to design the best formulation that will meet the requirements for therapeutic action at the sites of infection in the human body.

\section{Conclusions.}

Antibiotic therapy based on transcription factor decoys is a novel and specific approach to fight antimicrobial resistance by blocking essential genes in pathogenic bacteria. One of the main challenges associated with their use is the necessity of finding the right carrier to transfect them through the bacterial cell wall and to protect them from DNA-degrading enzymes, both in the biological fluids and in the bacterial cytoplasm. In this work, we have presented a new cationic bolaamphiphilic surfactant, [12-bis-THA] $\mathrm{Cl}_{2}$ which has proven to meet these requirements in vivo. We have investigated its behavior in aqueous solution, proving that it forms a mixture of vesicular and elongated nanostructured assemblies upon dissolution; the latter structure is the thermodynamically stable morphology into which the system converts entirely over time. Interestingly, storing the samples at $4{ }^{\circ} \mathrm{C}$ allowed us to trap the system in its metastable vesicular 
form. Next, we have evaluated its interaction with a model TFD, confirming the formation of nanoplexes: the main driving force for DNA condensation appears to be the electrostatic interaction between the oppositely charged species, the cationic bolaamphiphile and the polyanionic TFD backbone. The types of aggregates were independent on the morphology of the initial bolaamphiphile assemblies.

Importantly, we have demonstrated that this complex can dissociate in the appropriate conditions, for example in the presence of a competing anion such as taurocholate, to release the TFD in its biologically active form. These findings evoke the possibility to formulate the nanoplexes as smart, stimuli-responsive nanocarriers allowing the controlled release of the TFD. Further work will focus on the elucidation of additional aspects, like time and temperature stability of the complex, that still need to be clarified, in view of the design of [12-bis-THA]Cl 2 -based formulations that will accomplish transport, protection, transfection and release of TFDs to perform a successful therapeutic action.

${ }^{\S}$ Supporting Information (SI). Thermogravimetric analysis of pure [12-bis-THA $] \mathrm{Cl}_{2}$; surface tension $v s$. time plots of [12-bis-THA] $\mathrm{Cl}_{2}$ aqueous solutions; fluorescence emission spectra of [12bis-THA $] \mathrm{Cl}_{2}$ at different concentrations in aqueous solutions and relative UV-vis spectra; circular dichroism titrations of the TFD with [12-bis-THA $] \mathrm{Cl}_{2}$, and of a TFD/[12-bis-THA $] \mathrm{Cl}_{2}$ complex with sodium taurocholate, plus the relative absorption spectra, are supplied as Supporting Information.

Acknowledgments. This work was funded under the 7-People Framework - Marie Curie Industry and Academia Partnerships \& Pathways scheme (grant agreement nr. 612338). Cryo-TEM: Mrs. 
Ghislaine Frébourg (Institut de Biologie Paris-Seine, Université Pierre et Marie Curie, Paris,

France). Elemental analysis: Dr. Susanna Pucci and Dr. Sandra Cencetti, Laboratory of Microanalysis (University of Florence). ICP-AES: Dr. Mirko Severi and the research team headed by prof. Roberto Udisti (Department of Chemistry, University of Florence).

\section{References.}

[1] L.L. Silver, Challenges of Antibacterial Discovery, Clin. Microbiol. Rev. 24 (2011) 71-109. doi:10.1128/CMR.00030-10.

[2] D.J. Payne, M.N. Gwynn, D.J. Holmes, D.L. Pompliano, Drugs for bad bugs: confronting the challenges of antibacterial discovery, Nat. Rev. Drug Discov. 6 (2007) 29-40. doi: $10.1038 / \mathrm{nrd} 2201$.

[3] E. Leung, D.E. Weil, M. Raviglione, H. Nakatani, The WHO policy package to combat antimicrobial resistance, Bull. World Health Organ. 89 (2011) 390-392. doi:10.2471/BLT.11.088435.

[4] M. Kutateladze, R. Adamia, Bacteriophages as potential new therapeutics to replace or supplement antibiotics, Trends Biotechnol. $28 \quad$ (2010) 591-595. doi:10.1016/j.tibtech.2010.08.001.

[5] A. Extance, Biologics target bad bugs, Nat. Rev. Drug Discov. 9 (2010) 177-178. doi:10.1038/nrd3129.

[6] T.K. Lind, P. Zielińska, H.P. Wacklin, Z. Urbańczyk-Lipkowska, M. Cárdenas, Continuous Flow Atomic Force Microscopy Imaging Reveals Fluidity and Time-Dependent Interactions of Antimicrobial Dendrimer with Model Lipid Membranes, ACS Nano. 8 (2014) 396-408. doi:10.1021/nn404530z.

[7] B.L. Geller, Antibacterial antisense, Curr. Opin. Mol. Ther. 7 (2005) 109-113. PMID:15844617.

[8] M.J. Mann, V.J. Dzau, Therapeutic applications of transcription factor decoy oligonucleotides, J. Clin. Invest. 106 (2000) 1071-1075. doi:10.1172/JCI11459.

[9] M. McArthur, M.J. Bibb, Manipulating and understanding antibiotic production in Streptomyces coelicolor A3(2) with decoy oligonucleotides, Proc. Natl. Acad. Sci. 105 (2008) 1020-1025. doi:10.1073/pnas.0710724105.

[10] K. Komagata, K.-I. Suzuki, Lipid and cell-wall analysis in bacterial systematics. in: Methods in Microbiology, vol. 19, Academic Press Ltd., 1987.

[11] D. Simberg, D. Danino, Y. Talmon, A. Minsky, M.E. Ferrari, C.J. Wheeler, et al., Phase Behavior, DNA Ordering, and Size Instability of Cationic Lipoplexes: Relevance to Optimal Transfection Activity, J. Biol. Chem. 276 (2001) 47453-47459. doi:10.1074/jbc.M105588200.

[12] V. Weissig, J. Lasch, G. Erdos, H.W. Meyer, T.C. Rowe, J. Hughes, DQAsomes: A Novel Potential Drug and Gene Delivery System Made from Dequalinium ${ }^{\mathrm{TM}}$, Pharm. Res. 15 (1998) 334-337. doi:10.1023/A:1011991307631. 
[13] J. Lasch, A. Meye, H. Taubert, R. Koelsch, J. Mansa-ard, V. Weissig, Dequalinium TM Vesicles Form Stable Complexes with Plasmid DNA which Are Protected from DNase Attack, Biol. Chem. 380 (1999). doi:10.1515/BC.1999.080.

[14] V. Weissig, C. Lizano, V.P. Torchilin, Selective DNA Release from DQAsome/DNA Complexes at Mitochondria-Like Membranes, Drug Deliv. 7 (2000) 1-5. doi:10.1080/107175400266722.

[15] M. McArthur, Transcription factor decoys for the treatment and prevention of infections caused by bacteria including clostridium difficile. US Patent App. 13/802,103, 2013.

[16] H.C. van de Hulst, Light scattering by small particles, Dover Publications, New York, 1991.

[17] P.N. Pusey, Dynamic Light Scattering, in: Neutrons X-Rays Light Scatt. Methods Appl. Soft Condens. Matter, North Holland, Peter Lindner and Thomas Zemb, 2002.

[18] S.W. Provencher, A constrained regularization method for inverting data represented by linear algebraic or integral equations, Comput. Phys. Commun. 27 (1982) 213-227. doi:10.1016/0010-4655(82)90173-4.

[19] J. Ulmius, B. Lindman, G. Lindblom, T. Drakenberg, 1H, 13C, 35Cl, and 81Br NMR of aqueous hexadecyltrimethylammonium salt solutions: Solubilization, viscoelasticity, and counterion specificity, J. Colloid Interface Sci. 65 (1978) 88-97. doi:10.1016/00219797(78)90261-8.

[20] N. Nuraje, H. Bai, K. Su, Bolaamphiphilic molecules: Assembly and applications, Prog. Polym. Sci. 38 (2013) 302-343. doi:10.1016/j.progpolymsci.2012.09.003.

[21] L. Ziserman, H.-Y. Lee, S.R. Raghavan, A. Mor, D. Danino, Unraveling the Mechanism of Nanotube Formation by Chiral Self-Assembly of Amphiphiles, J. Am. Chem. Soc. 133 (2011) 2511-2517. doi:10.1021/ja107069f.

[22] R. Nagarajan, Self-assembly of bola amphiphiles, Chem. Eng. Commun. 55 (1987) 251-273. doi:10.1080/00986448708911931.

[23] K. Kalyanasundaram, J.K. Thomas, Environmental effects on vibronic band intensities in pyrene monomer fluorescence and their application in studies of micellar systems, J. Am. Chem. Soc. 99 (1977) 2039-2044. doi:10.1021/ja00449a004.

[24] J.R. Lakowicz, Principles of Fluorescence Spectroscopy., Third Edition, Springer, 2006.

[25] A. Memoli, L.G. Palermiti, V. Travagli, F. Alhaique, Effects of surfactants on the spectral behaviour of calcein (II): a method of evaluation, J. Pharm. Biomed. Anal. 19 (1999) 627632. doi:10.1016/S0731-7085(98)00229-5.

[26] F. Menger, S. Wrenn, Interfacial and Micellar Properties of Bolaform Electrolytes, J. Phys. Chem. 78 (1974) 1387-1390. doi:10.1021/j100607a600.

[27] S. Yiv, K.M. Kale, J. Lang, R. Zana, Chemical relaxation and equilibrium studies of association in aqueous solutions of bolaform detergents. 1. Dodecane-1,12bis(trimethylammonium bromide), J. Phys. Chem. 80 (1976) 2651-2655. doi:10.1021/j100565a006.

[28] J. Lasch, A. Hildebrand, Isothermic titration calorimetry to study cmes of neutral surfactants and of the liposome-forming bolaamphiphile Dequalinium ${ }^{\circledR}$., J. Liposome Res. 12 (2002) 51-56. doi:10.1081/LPR-120004776.

[29] W. Van de Sande, A. Persoons, The size and shape of macromolecular structures: determination of the radius, the length and the persistance length of rod-like micelles of dodecyldimethylammonium chloride and bromide, J. Phys. Chem. 89 (1985) 404-406. doi:10.1021/j100249a007.

[30] J.N. Israelachvili, Intermolecular and surface forces, 3. ed, Elsevier, Acad. Press, 2011. 
[31] V. Guida, Thermodynamics and kinetics of vesicles formation processes, Adv. Colloid Interface Sci. 161 (2010) 77-88. doi:10.1016/j.cis.2009.11.004.

[32] R. Saha, P.K. Verma, R.K. Mitra, S.K. Pal, Structural and dynamical characterization of unilamellar AOT vesicles in aqueous solutions and their efficacy as potential drug delivery vehicle, Colloids Surf. B Biointerfaces. 88 (2011) 345-353. doi:10.1016/j.colsurfb.2011.07.012.

[33] J. Kypr, I. Kejnovska, D. Renciuk, M. Vorlickova, Circular dichroism and conformational polymorphism of DNA, Nucleic Acids Res. 37 (2009) 1713-1725. doi:10.1093/nar/gkp026.

[34] V.M. Jadhav, R. Valaske, S. Maiti, Interaction Between 14mer DNA Oligonucleotide and Cationic Surfactants of Various Chain Lengths, J. Phys. Chem. B. 112 (2008) 8824-8831. doi:10.1021/jp8017452.

[35] T. Zhou, G. Xu, M. Ao, Y. Yang, C. Wang, DNA compaction to multi-molecular DNA condensation induced by cationic imidazolium gemini surfactants, Colloids Surf. Physicochem. Eng. Asp. 414 (2012) 33-40. doi:10.1016/j.colsurfa.2012.08.060.

[36] Z. Pietralik, R. Krzysztoń, W. Kida, W. Andrzejewska, M. Kozak, Structure and Conformational Dynamics of DMPC/Dicationic Surfactant and DMPC/Dicationic Surfactant/DNA Systems, Int. J. Mol. Sci. 14 (2013) 7642-7659. doi:10.3390/ijms14047642.

[37] D. Luo, M.W. Saltzman, Synthetic DNA delivery systems., Nat. Biotechnol. 18 (2000) 3337. doi: $10.1038 / 71889$.

[38] B. Sohrabi, V. Khani, A.A. Moosavi-Movahedi, P. Moradi, Investigation of DNA-cationic bolaform surfactants interaction with different spacer length, Colloids Surf. B Biointerfaces. 110 (2013) 29-35. doi:10.1016/j.colsurfb.2013.04.032.

[39] M. Khan, C.Y. Ang, N. Wiradharma, L.-K. Yong, S. Liu, L. Liu, et al., Diaminododecanebased cationic bolaamphiphile as a non-viral gene delivery carrier, Biomaterials. 33 (2012) 4673-4680. doi:10.1016/j.biomaterials.2012.02.067.

[40] K. Kunath, Low-molecular-weight polyethylenimine as a non-viral vector for DNA delivery: comparison of physicochemical properties, transfection efficiency and in vivo distribution with high-molecular-weight polyethylenimine, J. Controlled Release. 89 (2003) 113-125. doi:10.1016/S0168-3659(03)00076-2.

[41] E.C. Long, J.K. Barton, On demonstrating DNA intercalation, Acc. Chem. Res. 23 (1990) 271-273. doi:10.1021/ar00177a001.

[42] C. Boulanger, C. Di Giorgio, J. Gaucheron, P. Vierling, Transfection with Fluorinated Lipoplexes Based on New Fluorinated Cationic Lipids and in the Presence of a Bile Salt Surfactant, Bioconjug. Chem. 15 (2004) 901-908. doi:10.1021/bc049942+. 